\title{
From tumour perfusion to drug delivery and clinical translation of in silico cancer models
}

\author{
Myrianthi Hadjicharalambous ${ }^{\mathrm{a}}$, Peter A. Wijeratne ${ }^{\mathrm{b}}$, Vasileios \\ Vavourakis ${ }^{\mathrm{a}, \mathrm{c}, *}$ \\ ${ }^{a}$ Department of Mechanical \& Manufacturing Engineering, University of Cyprus, \\ Nicosia, Cyprus \\ ${ }^{b}$ Centre for Medical Image Computing, Department of Computer Science, University \\ College London, London, UK \\ ${ }^{c}$ Department of Medical Physics $\&$ Biomedical Engineering, University College London, \\ London, UK
}

\begin{abstract}
In silico cancer models have demonstrated great potential as a tool to improve drug design, optimise the delivery of drugs to target sites in the host tissue and, hence, improve therapeutic efficacy and patient outcome. However, there are significant barriers to the successful translation of in silico technology from bench to bedside. More precisely, the specification of unknown model parameters, the necessity for models to adequately reflect in vivo conditions, and the limited amount of pertinent validation data to evaluate models' accuracy and assess their reliability, pose major obstacles in the path towards their clinical translation. This review aims to capture the state-of-the-art in in silico cancer modelling of vascularised solid tumour growth, and identify the important advances and barriers to suc-
\end{abstract}

*v.vavourakis@ucl.ac.uk

Email addresses: hadjicharalambous.myrianthi@ucy.ac.cy (Myrianthi

Hadjicharalambous), p.wijeratne@ucl.ac.uk (Peter A. Wijeratne),

v.vavourakis@ucl.ac.uk; vavourakis.vasileios@ucy.ac.cy (Vasileios Vavourakis) 
cess of these models in clinical oncology. Particular emphasis has been put on continuum-based models of cancer since they - amongst the class of mechanistic spatio-temporal modelling approaches — are well-established in simulating transport phenomena and the biomechanics of tissues, and have demonstrated potential for clinical translation. Three important avenues in in silico modelling are considered in this contribution: first, since systemic therapy is a major cancer treatment approach, we start with an overview of the tumour perfusion and angiogenesis in silico models. Next, we present the state-of-the-art in silico work encompassing the delivery of chemotherapeutic agents to cancer nanomedicines through the bloodstream, and then review continuum-based modelling approaches that demonstrate great promise for successful clinical translation. We conclude with a discussion of what we view to be the key challenges and opportunities for in silico modelling in personalised and precision medicine.

Keywords: multiscale, personalized models, precision medicine, solid tumor simulation, angiogenesis, drug transport

\section{Introduction}

Cancer is regarded a complex and heterogeneous disease [1] that encompasses a large family of pathologies that involve abnormal cell growth and death, establishment of new blood vessels, and the potential to invade or spread to other parts of the body. Specifically, solid tumour formation involves the co-evolution of neoplastic cells together with extracellular matrix, tumour vasculature and immune cells. Moreover, tumour development is orchestrated by multiple biological interactions and events occurring in the 


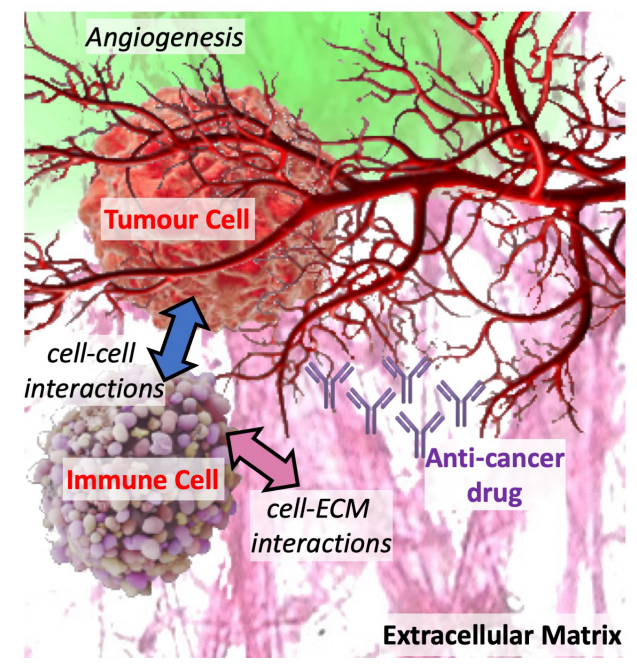

Figure 1: Targeting cancer requires elucidating the complexities of the tumour-host microenvironment (THM), particularly the interactions between cancerous and host cells (including immune), cells and the matrix, the role of the microvasculature and the mechanisms involved in the transport of therapeutic drugs.

molecular domain among individual genes and proteins, and at the realm of cells - between functionally diverse cells and tissues. Malignant tumour formation is caused by genetic and epigenetic alteration and deregulation of the molecular pathways (e.g., motility, proliferation, differentiation, and cell death) that normally maintain the physiological properties of eukaryotic cells. Also, locally-activated components of the host microenvironment (cells and their surrounding extracellular matrix) in turn influence the phenotypic behaviour of tumour cells, i.e. their proliferative and migratory potential. Thus, understanding cancer progression requires the elucidation of collective properties of cells within a tissue and their interaction within the microenvironment of the host [2,3], see Figure 1.

From the cancer therapy perspective, effective delivery of a cancer drug 
through the systemic circulation requires thorough understanding of the tumour patho-physiology, which includes the micro-circulatory and the immune system [4]. As a result, and in view of the dynamic environment of a developing - or regressing under the impact of therapy - malignant tumour, further investigation of the salient characteristics of the tumour vasculature and its unique biophysical markers is required.

In the recent years, with the emergence of precision and personalised medicine, cancer researchers have driven the development of new methods for systems analysis of the disease and its treatment. ${ }^{1}$ In this regard, reductionist in vitro and in vivo experimental models have been widely used to investigate the tumour-host microenvironment (THM) heterogeneity along with the factors that influence the effectiveness of chemotherapeutic agents and radiotherapy (e.g. that includes the size, charge and solubility drugs, the dosing level and schedule, the pharmacokinetics of drug residence in the circulation, the pharmacodynamics of cell killing in response to drug exposure) $[5,3,6]$. However, due to the plethora of factors to consider, comprehensive system biology approaches are more appropriate for investigating potential treatment strategies compared to experimental models alone. As such, it is now becoming apparent that reliable and successful personalised treatment necessitates an integrative, multiscale, systems biology approach to understand cancer, and therefore interrogate and predict the patho-physiology of the disease.

In the last decades, and in parallel to in vitro and in vivo research, in

\footnotetext{
${ }^{1}$ https://www . cancer.gov/about-cancer/treatment/types/precision-medicine
} 
silico models are gaining significant recognition for their potential to predict patient-specific outcomes to cancer therapy. ${ }^{2}$ In silico cancer modelling used to refer collectively to applied mathematical and computational methods in modelling cancer - can prove instrumental to aid our understanding of the THM mechano-biology, drug design and testing, and the treatment of complex biological systems [7] - with cancer being no exception to this. Specifically, mathematical modelling of cancer dates back to the first quarter of the 20th century - a historical treaty of the topic is covered in the review paper by Araujo and McElwain [8] - while computational modelling has seen rapid developments with the advent of improved computing power. In silico approaches can be invaluable for testing hypotheses, falsifying theories and identifying biological mechanisms in cancer. Contrary to in vitro and in vivo approaches, in silico models can permit interrogating the disease at multiple spatio-temporal scales $[9,10]$, thus offering an effective way to discover new drugs for cancer therapy in clinic.

Integrating heterogeneous dynamic data into quantitative in silico cancerpredictive models holds great promise to significantly increase our ability to understand and rationally intervene in disease-perturbed biological systems $[11,12]$. At the same time, by incorporating data from individual patients (e.g. medical images of tumour anatomy and evolution, angiography images of tumour vasculature), in silico models allow for personalised model predictions as well as testing of potential treatments on a patient-specific basis. In addition, in silico cancer models can enable the description of interactions

\footnotetext{
${ }^{2}$ https://www.fda.gov/science-research/about-science-research-fda/ how-simulation-can-transform-regulatory-pathways
} 
in the THM that are also amenable to quantitative (in vivo or ex vivo) measurements. Furthermore, with the advent of high-throughput experimental tools, cancer researchers are able to simultaneously measure thousands of biomolecules [13], hence, paving the way for in silico model construction of increasingly large and diverse systems of cancer mechano-biology $[14,15]$.

The purpose of this contribution is neither to review the entire scientific field of in silico cancer modelling, nor to attempt being all-inclusive of cancer mechano-biology and drug design / discovery; broader surveys in cancer modelling are provided in $[16,17,18]$. In this review we outline recent applications of in silico models in cancer with particular emphasis on solid tumour angiogenesis and cancer drug delivery models - both critical elements in improving our understanding of the factors limiting the delivery of chemotherapeutics and maximising the efficacy of chemotherapy and cancer treatments. We finish with an overview of recent examples of clinical translations of continuum-based cancer models, with an emphasis on applications on in silico testing of potential therapies, and a discussion about the means for model validation.

Briefly, as it will be discussed in Section 2, angiogenesis provides a means for supplying nutrients and oxygen to a 'starving growing tumour', constituting a major event in cancer progression and as such an important therapeutic target against the disease $[19,20]$. At the same time, the newly-formed vasculature is pathological and suffers from poor perfusion, impeding drug delivery (Section 3) and the efficacy of chemotherapy. Therefore, in silico models of tumour-induced angiogenesis and drug delivery could play a key role in providing a mechanistic understanding of the factors improving or hindering 
drug delivery and, accordingly, in suggesting optimised therapeutic avenues. From the wide variety of available in silico models on angiogenesis and drug delivery, in this review we narrow our attention to continuum-based models and multiscale models - detailed classification of cancer models is provided in the survey [21]. In particular, by focusing on large-scale behaviours, continuous models have the ability to be largely specified by in vivo imaging data, such as angiography images of tumour vasculature, and magnetic resonance (MR) and ultrasound images of tumour geometry. Accordingly, the accuracy of continuum-based models can be enhanced by informing the model using in vivo data, while data can also serve as a basis for model validation. Exploiting the obvious potential for use of in silico models in the clinic, we further examine recent studies into potential avenues for the clinical translation of in silico tools to basic and clinical cancer research (Section 4). Finally, we conclude with the main obstacles and challenges in in silico cancer modelling (Section 5) along with the opportunities for its future use as a non-invasive personalised clinical tool.

\section{Angiogenesis models}

Angiogenesis is a complex process, representing the formation of a new network of blood vessels from pre-existing vasculature. Despite its vital role in physiological phenomena such as wound healing and placenta formation in the embryonic stage, angiogenesis is more commonly associated with pathological conditions such as arthritis, diabetic retinopathy and notably, cancer [22]. Indeed, angiogenesis has been linked to cancerous growth, invasion and

metastasis and it represents a key event in tumour development [18, 23, 19]. 
As first proposed by Folkman in the early 1970s [24], the newly formed vasculature provides nutrients and oxygen to the tumour enabling the viability of malignant cells, which are then able to enter the circulatory system through the tumour vasculature and migrate to distant body tissues [25, 26]. Accordingly, angiogenesis marks the transition from the dormant avascular tumour growth to an aggressive, metastatic and potentially fatal phase, constituting a pivotal stage in cancer development and, thus, a significant therapeutic avenue [20, 27].

The highly complex process of angiogenesis requires a well-orchestrated cascade of heterogeneous events initiated by the release of chemical substances, termed tumour angiogenic factors (TAFs), by tumour cells [28]. The formation of the new vasculature is largely guided by chemotaxis, with the endothelial cells (ECs) which line the vasculature walls moving towards higher TAF concentrations. At the same time, ECs' movement is also markedly affected by haptotaxis, with the cells moving towards regions with higher concentration of matrix-bound proteins such as fibronectin [29], as well as by mechanotaxis, with ECs affected by mechanical stresses' gradients [30]. Detailed description of angiogenesis can be found in the in-depth works $[19,31,32]$. Notably, tumour-induced angiogenesis results in a dense, tortuous and chaotic vascular network [33, 34], which although pathological, is able to satisfy the tumour's metabolic needs as well as provide a means for its metastasis. Nevertheless, the newly developed network is often associated with hypo-perfusion, hindering the delivery of chemotherapeutics and, therefore, reducing treatment efficiency [35, 36].

Due to its crucial role in cancer development, along with its adverse ef- 
fects on the efficacy of chemotherapy, angiogenesis has received significant research interest, with the ultimate goal of developing anti-angiogenic treatments. Despite a plethora of experimental studies which have significantly improved our understanding of the angiogenesis process [24, 28, 29], important phenomena occurring at several spatio-temporal scales remain poorly understood. To this end, a wide range of in silico mathematical models have been proposed, aiming in providing a mechanistic understanding of the angiogenesis process, while bypassing the need for expensive and time consuming experiments. Existing angiogenesis models present substantial differences, varying in terms of their complexity or level of abstraction, the spatial and temporal scales described and the physical phenomena included into the model. Additionally, models of angiogenesis are commonly classified with respect to their mathematical representation of important model variables into two main categories: continuum-based models, which capture the large-scale behaviour of the system and treat the vascular density and concentration of chemical species as continuous quantities, and discrete cell-based models which focus on individual cell behaviour and cell-cell interactions and allow individual cells to move, proliferate and metastasise based on defined rules.

The focus of this section will be on continuum-based angiogenesis models which can be informed and tuned using routinely-acquired imaging data, e.g. angiography images, ultrasound, elastography data, or magnetic resonance images of the tumour anatomy. The interested reader can refer to Mantzaris et al. [37] and Scianna et al. [38] for broader and more detailed reviews of both continuous and discrete mathematical modelling of angiogenesis, and to Magi et al. [18] for a description of angiogenesis modelling from the cell 
and genome perspective.

\subsection{Continuous models}

Continuous models of angiogenesis rely on the hypothesis that the system modelled is continuous, with neighbouring regions behaving similarly or presenting gradual spatial variations. Within this framework, the molecular and cellular structure of the tumour and healthy tissue are disregarded and focus is placed on macroscopic quantities such as cell densities and concentrations of chemical substances which are described by continuous fields. Additionally, the structure of the vasculature network is not explicitly described, and therefore new vascular growth is modelled as an increase of the vascular density. Commonly, continuous models employ a set of advectionreaction-diffusion equations to describe the dynamics of cell density $\rho$ and chemical concentration $c$, which in their general form is given by:

$$
\begin{aligned}
& \frac{\partial \rho}{\partial t}+\boldsymbol{\nabla} \cdot(\rho \mathbf{v})=\boldsymbol{\nabla} \cdot(K \boldsymbol{\nabla} \rho)+f(\rho, c), \\
& \frac{\partial c}{\partial t}+\boldsymbol{\nabla} \cdot(c \mathbf{u})=\boldsymbol{\nabla} \cdot(D \boldsymbol{\nabla} c)+g(\rho, c),
\end{aligned}
$$

where $c$ denotes the concentration of all chemical substances and ECMrelated components assumed to affect the angiogenesis process (e.g. TAFs such as vascular endothelial growth factor (VEGF) and basic fibroblast growth factor (bFGF), or matrix-bound proteins such as fibronectin and laminin). Similarly, $\rho$ describes the densities of all cell types involved in the model;

for instance, ECs and tip or stalk endothelial cells (TECs and SECs, respectively). Function $f$ models cell proliferation and apoptosis while function $g$ the production, uptake and decay of the chemical factors, respectively, and 
they depend on the densities of all cell types considered and the concentration of all chemical substances modelled. Additionally, $K$ and $D$ denote the diffusion coefficients and $\mathbf{v}$ and $\mathbf{u}$ are the advection (velocity) vectors corresponding to cell types and chemical substances, respectively. The advection velocity $\mathbf{v}$ is most commonly directed towards the hypoxic regions through the use of a chemotactic term, and towards regions with higher ECM concentration in models also accounting for haptotaxis.

Mathematical models of angiogenesis that describe the vascular density as a continuous field date back to the pioneering work of Balding and McElwain [39] in 1985. Motivated by models of fungal growth, this seminal work can capture the phenomena of branching and anastomosis through separate differential equations for the TECs and SECs density - the former cells are leading the new vessel generation, while the latter are facilitating capillary growth by forming the rear of a new lumen through cell division. This model is based on the snail-trail concept, namely that TECs guide the generation of new vessels by chemotactically responding to TAF stimuli, whereas the evolution of SECs is proportional to the flux of TECs. Following this approach, Byrne and Chaplain presented a one-dimensional model which also accounted for the dynamics of the TAF concentration [40]. Despite its simplified structure, this model was able to reproduce experimentally observed behaviour and to provide valuable insights into the angiogenesis process, such as suggesting that the balance between TECs proliferation, decay and TAF concentration affects the formation of new vasculature.

While early models focused on the chemotactic response of cells to TAFs, subsequent modelling efforts also accounted for the haptotactic effects of 
matrix-bound proteins on ECs. Prominent examples include the works of Orme and Chaplain [41] as well as that of Anderson and Chaplain [42], in which EC migration is dependent on the concentration gradient of both TAFs and fibronectin. These models highlight that haptotaxis is essential to achieve angiogenesis in a tumour site and produced more realistic two-dimensional simulations, enabling qualitative comparisons with in vivo experiments. An important extension of this model was proposed by Holmes and Sleeman, whereby the ECs movement is also influenced by mechanical aspects [43]. Building on the mechanical model of Manoussaki et al. for in vitro formation of vascular networks [44], they incorporated the effect of traction forces exerted from the ECs on ECM fibres during ECs' movement which, in turn, influences the cells' direction of migration. The cell densities and concentrations of fibronectin and TAFs are treated as in earlier continuous models, while the ECM is modelled as a viscoelastic material. A similar mechanical model was also used by Murray to describe the interactions between cells and the viscoelastic ECM during pattern formation of vascular networks [45].

Within the same continuum-based modelling framework, more elaborate models were developed by Levine et al. [46], which consider additional chemical substances as well as angiogenic inhibitors and proteolytic enzymes. This series of studies started with a simple model describing the onset of angiogenesis and considered the mechanism through which ECs convert TAFs into proteases, which in turn can degrade the basal lamina - part of the changes occurring prior to the formation of new vasculature. The model was then extended to consider the regulating role of macrophages and pericytes in the spatio-temporal course of angiogenesis, as well as to investigate the ac- 
tion of anti-angiogenic factors such as angiostatin [47]. Further extension of these works resulted in a complex five-species model considering ECs, TAFs, preoteases, fibronectin, and angiostatin, which presented good agreement with experimental observations of growth rates. All of these works are based however on the theory of reinforced random walks for the movement of cell populations and employ the Michaelis-Menten model to describe enzyme kinetics.

An important enhancement of the continuous models of angiogenesis was the coupling to tumour growth processes. De Angelis and Preziosi [48] proposed one of the earliest coupled models, whereby the evolution of the tumour under the influence of angiogenesis was formulated as a free-boundary value problem. The model considered the densities of living and dead tumour cells, nutrients, new capillaries, TAFs and growth inhibitory factors. The coupled model was able to predict experimentally observed changes in tumour morphology (e.g. necrotic core, proliferative outer rim), as well as the regression of capillaries under the influence of anti-angiogenic factors. Hogea et al. [49] employed the level set method to solve the De Angelis model and were able to capture the avascular and vascular phase of tumour growth along with the time of transition. An alternative model describing the vascular tumour growth was recently proposed by Kremheller et al. [50], employing a continuum-based multi-phase model relying on the thermodynamically constrained averaging theory. This model considers five different phases: ECM, treated as a porous solid phase, tumour cells, host cells and the interstitial fluid forming three fluid phases, and the neovasculature modelled as a separate phase. The proposed multi-phase approach enabled interactions between 
ECM, flows and angiogenesis and was able to reproduce increased interstitial pressure in tumours, which is an important factor in drug delivery efficacy. Additionally, Voutouri et al. [51] recently proposed a continuum-based approach focusing on vessel co-option, whereby tumour progression is sustained without angiogenesis, with tumours receiving nutrients by the existing vasculature. This work introduced a model of secondary angiogenesis - occurring as a result of co-option-induced hypoxia - coupled with a biomechanical model of tumour growth, in which the tumour is treated as a biphasic material. Their model was employed to test anti-angiogenic and anti-co-option treatments, suggesting that vessel co-option should be targeted in combination with angiogenesis.

Finally, a recent work by Hormuth et al. [52] aimed at modelling tumour growth and angiogenesis at the tissue level, relying on in vivo magnetic resonance imaging (MRI). In particular, diffusion-weighted MRI and dynamic contrast-enhanced MRI data provided tumour and blood volume fractions, which were used to initialize, personalise and calibrate the proposed model. The predictions of eight personalised models presented good agreement with MRI data in terms of tumour growth and blood volume predictions, highlighting the potential of image-based in silico modelling to assist in patientspecific treatment strategies.

\subsection{Models employing the phase-field approach to capture the vasculature}

The above-mentioned continuous models have facilitated a better understanding of the complex processes involved in angiogenesis and of the influence of individual angiogenesis features on the formation of the new vasculature. Nevertheless, continuous models are unable to predict detailed 
features of the morphology of vascular structures and capture heterogeneities in the tumour vascular network. Accordingly, direct comparisons with experimental angiogenesis data are not possible, hindering model validation and improvement. Furthermore, by lacking the ability to predict the morphology of the vasculature, purely continuous models are unable to model and investigate the blood flow through the vasculature, along with the transport of nutrients and drugs to the tumour site - important model aspects which could pave the way for improved drug delivery.

Travasso and colleagues [53] proposed an alternative approach to alleviate this issue, by adopting the phase-field method. Specifically, the proposed model introduces an order variable which identifies the regions of the domain occupied by capillaries. The model is able to capture the chemotactic response of ECs and their proliferation, and investigates how the final vascular configuration is affected by important model parameters; for instance, higher levels of angiogenic factors lead to increased branch density and higher vessel diameters. Extending this work, Santos-Oliveira et al. [54] developed a continuous model of sprouting angiogenesis which investigated the influence of the elastic properties of the tissue on the morphology of the formed sprouts. They also considered cell-cell adhension forces and traction forces exerted by the TECs and concluded that the strain on TECs induced by tension triggers cells proliferation in the SECs that follow. Importantly, they arrived at these conclusions using a compact model with a reduced number of parameters, incurring low computational cost.

Another example of the phase-field approach was the work of $\mathrm{Xu}$ et al. [55], which modelled both tumour growth and tumour-induced angiogenesis. 
Notably, the authors use the geometry of a human colon extracted from a histo-pathological image as their computational domain, aiming to provide physiologically relevant results in a realistic setup.

Following the phase-field approach for capturing the vascular structure, Villanova et al. [56] investigated important stages throughout the angiogenesis process, namely vascular growth, regression and regrowth. Employing a discrete agent-based model for the TECs, they were able to model the lateral inhibition mechanism, whereby ECs which neighbour TECs are prevented from also becoming TECs. They later extended this work to include porous media fluid flow with the aim of understanding the role of flow in tumour angiogenesis, and suggested that the interstitial flow might contribute to tumour malignancies and ineffective cancer treatments [57]. Using a similar hybrid approach, Moreira-Soares et al. [58] introduced an additional order parameter to describe the distribution of hypoxic tissue cells and concentration of growth factors and showed that these factors significantly influence the morphology of the neovasculature and promote vessel anastomosis. Importantly, the works considered in this section have shown good agreement with in vivo experiments and provided valuable insight that can be of use for anti-angiogenic treatment design.

\subsection{Continuous models employing a discrete vasculature description}

An alternative approach employed for capturing morphological features of vascular networks throughout the angiogenesis process was to explicitly include a discrete description of the vasculature in terms of line segments or point masses. Sun et al. [59, 60] introduced the concept of a discrete description of sprout tips, moving as point masses under the influence of contin- 
uum fields of chemotactic and haptotactic factors. This so called discrete-incontinuum model was able to realise branching and anastomosis phenomena, as well as sufficiently reproduce the dendritic capillary network structure observed in vivo. An extension of this model to three dimensions was then proposed by Milde et al. [61], whereby a particle representation was used for individual TECs. This model was used to assess the influence of the ECM structure on the vessel morphology and TECs' migrating speed, with the ECM modelled as a collection of randomly distributed fiber bundles.

Focusing on the vasculature, a series of papers by Zheng et al. , employed an explicit representation of capillaries, instead of cells [62, 63, 64], whereby the capillaries were treated as one-dimensional viscoelastic cylinders, with constant radius. The proposed viscoelastic model was initially able to investigate the mechanisms of capillary extension with and without proliferation, suggesting that SECs' proliferation is essential for supporting the TECs' driven capillary extension [63]. The model was subsequently extended to examine the effect of several growth factors and angiopioetins on proliferation, migration and maturation of ECs as well as investigate the influence of antiangiogenic therapies on the formation of new vasculature [62]. The model was then coupled with a tumour growth model, with the tumour following Darcy's law, which was used to investigate the efficiency of anti-angiogenic treatments aiming in VEFG inhibition [64]. Their simulations suggested the potential of such treatment to cause vessel regression and tumour shrinkage, but highlighted the importance of vessel normalization in treatment efficacy.

Following the modelling concepts of Sun et al. [59] and Milde et al. [61], whereby sprout tips are regarded as point masses moving in a continuum sub- 
stratum, Vavourakis et al. [65] proposed a more detailed angiogenesis model. This work presented a dynamic multiscale model of capillary growth, coupled with tumour growth, blood flow through the vasculature and interstitial flow, as well as remodelling of the ECM and the vasculature. In the developed three-dimensional model, the direction of elongation of individual TECs was affected by chemotactic and haptotactic factors as well as by the contribution of solid stresses (mechanotaxis). This elaborate model was informed by in vivo and in vitro data to produce physiologically accurate behaviours, and was thus able to reproduce the vascular density and distribution observed in optical frequency domain imaging scans of murine mammary adenocarcinomas as well as the experimental observation that growth-induced stresses affect the vascular morphology. Importantly, this study's results supported the hypothesis that the spatial arrangement of the newly formed vasculature is primarily affected by the stresses in the tumour rather than the interstitial fluid pressure, and as a consequence, by the biomechanics of the THM.

\section{Drug delivery models}

Drug delivery is the process whereby a foreign agent is introduced into a biological system in order to alter its behaviour. In terms of chemotherapy, the agent is a drug, and the delivery medium is usually the blood stream via intravenous injection. At the macroscale, the subsequent transport of the drug to the target site is governed by a series of physical processes: intravascular flow, which describes the motion of the drug within the host vasculature (e.g. blood vessels and capillaries); transvascular flow, which describes motion between the vasculature and the surrounding tissue; and interstitial flow, 
which describes the motion in the surrounding tissue.

Once at the target site, distribution and uptake of the drug is determined by its pharmacokinetics, which depends on the host biophysical environment, and its pharmacodynamics, which conversely determine how the drug affects its environment. The transport of chemotherapeutic agents can be modelled at multiple scales; from molecular dynamics at the atomic scale $\left(10^{-10} \mathrm{~m}\right)$ through stochastic-continuum models at the nano-/microscale $\left(10^{-9} \mathrm{~m}-10^{-6}\right.$ $\mathrm{m})$ to continuum-based models at the tissue or organ-scale $\left(10^{-3} \mathrm{~m}-10^{-1}\right.$ $\mathrm{m})$. Here we focus on continuum-based models of intravenous delivery of micro- and nanoscale drugs, and hence ignore both molecular-scale pharmacokinetics such as biomolecular corona formation [66], endocytosis [67], and macroscale drug properties such as geometry and polymer-specific effects [68]. For a review of models across multiple scales, see for example [21], and for general reviews of computational drug delivery see e.g. [4, 69].

In the most general terms, the total concentration of drug particles, $c$, in the blood stream at time $t$ can be modelled by the continuity equation:

$$
\frac{\partial c}{\partial t}=\boldsymbol{\nabla} \cdot(D \boldsymbol{\nabla} c)-\boldsymbol{\nabla} \cdot(\boldsymbol{v} c),
$$

where $\boldsymbol{v}$ is the mean blood velocity, and $D$ is the diffusion coefficient that is specified by the properties of the blood and drug particles. The spatial derivatives can be simplified in the special case of an incompressible fluid with constant diffusivity, which are reasonable assumptions for blood plasma as it is mainly comprised of water. The right-hand-side of the above equation is split into two terms that govern mass transport in blood: diffusion, which describes the mean Brownian motion of particles along a potential energy gra- 
dient, and convection, which describes the motion of particles in a stationary flow. The relative balance of the diffusion and convection terms depends on the particles' properties and the local blood velocity; for small particles (e.g.

oxygen), diffusion dominates for all but the highest blood velocities $\left(>10^{-3}\right.$ $\mathrm{cm} / \mathrm{s}$ ), and Equation 3 essentially becomes Fick's law of steady-state diffusion [70]; while for large particles (e.g. liposomes, nanoparticles) convection dominates for all but the lowest blood velocities $\left(<10^{-7} \mathrm{~cm} / \mathrm{s}\right)$ [4]. Therefore, the properties of the drug which are known a priori can be used to pose suitable models of intravascular, transvascular and interstitial flow. The subsequent uptake can then be modelled by a system of equations that are coupled to the local flow dynamics, which can be a function of measurable tissue properties such as permeability and heterogeneity.

\subsection{Compartmental models}

A compartmental approach to drug delivery modelling in cancer was first proposed by [71], where a two-compartment pharmacokinetic model of coupled ordinary differential equations was used to model the drug concentration in tumour tissue and blood plasma. The authors extended this approach to a three-compartmental model of coupled reaction-diffusion equations, allowing spatial drug concentration profiles to be modelled [72, 73]. Though several simplifying assumptions were made in both models (such as constant and homogeneous permeability), they were able to predict that drug concentration was highly sensitive to vascular density and permeability; factors which had already been identified as having a strong influence on drug delivery efficacy in vivo [74]. In all these models only drug concentration was predicted, and no attempt was made to describe the effect of the drug on cell growth 
(cancerous or otherwise). Furthermore, there were no description of the host tissue or vasculature and their interaction with drug delivery.

\subsection{Avascular models}

A mathematical model of solid tumour growth in the presence of inhibitors was formulated by [75], who described the tumour radius in 1D by an integro-differential equation, and the concentration of nutrients (e.g. oxygen, glucose) and inhibitors (e.g. immune cells) by two reaction diffusion equations. While not technically a model of drug delivery, it explicitly modelled necrosis and hence allowed the effect of different types of growthmodifying agents on solid tumour growth to be investigated. A similar approach was taken by Jackson and Byrne [76], who described solid tumour growth as a spherically-symmetric mixture of different cancer cell species coupled with a reaction-diffusion model of drug concentration. This model allowed for predictions of the drug concentration inside and outside the tumour, and the effect of cells with varying resistance on the time of regression. A cylindrically-symmetric model was studied by Bertuzzi et al. [77] to represent tumour growth and treatment in a confined environment (e.g. mammary ducts), using a system of coupled reaction-diffusion equations to describe the spatio-temporal distributions of cell, nutrient and drug concentration. The model allowed the authors to test the sensitivity of cells to oxygenation levels before and after treatment. An avascular model of multi-cellular tumour spheroid growth that coupled cell, nutrient and drug concentrations was proposed by Ward and King [78]. Their model predicted that multi-cell tumours were more resistant to therapy than mono-cell tumours, reflecting the pre-

dictions in [76] but with the additional effect of naturally varying cell types 
(e.g. active, quiescent, necrotic) as a result of nutrient concentration. However, none of these models describe the effect of vessel topology, or, crucially, the pathological development of new vessels (angiogenesis) as a result of the presence of tumour-associated angiogenic growth factors.

\subsection{Vascular models}

Modelling the effects of biophysical fields, such as chemotactic, haptotactic and mechanotactic gradients, is instrumental in providing a realistic description of the vasculature local to the tumour and biologically relevant conditions for drug delivery. In all the following models, unless stated explicitly, blood is treated as a Newtonian fluid. A 2D continuum-based model of tumour-induced blood vessel network growth coupled with blood and drug flow dynamics was proposed by McDougall et al. [79]. Here the angiogenesis model was comprised of a Fick-like diffusion equation governing vessel cell density as a function of chemo- and haptotaxis coupled with partial differential equations for the drug and fibronectin concentrations, and drug transport through the network was described by a Poiseuille constitutive model. This approach was extended to 3D by Stephanou et al. [80], where the authors used the model to study the effects of altering the vessel network's properties (such as density and structure), with implications for therapeutic approaches that target both solid tumours and the surrounding vasculature. In both models the vascular wall was assumed impermeable, and hence delivery was possible only at vessel outlets, and reaction kinetics were not modelled by the uptake function. Furthermore, vessels were assumed static, which is a reasonable assumption with respect to the much shorter timescale of drug delivery, but prevents realistic analysis of multiple sequential delivery times. There was 
also no account for interstitial fluid dynamics, which - in conjunction with the assumption of constant vessel permeability - precluded any analysis of the effect of drug size on delivery efficacy.

To investigate the effect of vessel permeability and drug size on drug transport and spatio-temporal distribution, Decuzzi et al. [81] used the Taylor-Aris theory of shear dispersion, which is a special case of Equation 3, to model nanoparticle concentration in 1D, and described intravascular flow and perfusion using Poiseuille and Darcy constitutive models, respectively. The model facilitated study of size-dependent transport and comparison between permeable and non-permeable vessels, which allowed the authors to make predictions of the threshold nanoparticle size for diffusive and convective transport. Apart from being restricted to one dimension, the model also assumed vessels as straight tubes, and was valid only in the case of long times after injection, in accordance with the Taylor-Aris theory. A similar approach was taken in [82], where the authors applied a Casson fluid law to describe blood flow. The authors found that both blood rheology and vessel permeability have an effect on diffusion, though again predictions were limited to 1D. A combined pharmacokinetic reaction-diffusion model of vascularised drug delivery in 2D was proposed by Sinek and colleagues [83], who studied the effects of nutrient, cell and drug heterogeneity. They found that all three have a large effect on delivery efficacy, and hence argued that macroscopic factors must be considered in conjunction with genetic factors when planning therapeutic interventions. These predictions, however, were limited to monolayer assays due to the two-dimensional nature of the model.

To make in silico predictions more relevant to in vivo conditions, 3D 
models of vascularised delivery have been proposed. In [84], a two compartmental model of tracer concentration combined with a reaction-convectiondiffusion model and a Darcy model of interstitial fluid pressure (IFP) were used to study the effect of heterogeneous vasculature on interstitial transport within a solid tumour. They predicted that tracer uptake was proportional to vessel leakiness (permeability), and inversely proportional to interstitial permeability. Interestingly, the model boundary conditions were specified using T1 weighted MRI data from an in vivo mouse sarcoma. A coupled 3D continuum-discrete model of nanoparticle delivery in the lung was proposed by [85]. The pulmonary tissue and capillary bed were described as separate porous continua, and a vascular graph model (VGM) was used to describe the drug spatio-temporal distribution at the discrete level, which was coupled to the continuum model via volume averaging theory. Simulations were performed to demonstrate the model, but no comparison was made with data, there was no tumour model, and the network was assumed static. Another continuum-discrete model of nanoparticle delivery was proposed by Tan et al. [86], who used a Brownian model of nanoparticle dynamics coupled with a continuum model of tissue mechanics. They used their model to study the effects of nanoparticle size, shear rate and vessel geometry on drug binding capabilities. Also, Thurber and Wittrup [87] proposed a simple mechanistic model of antibody uptake and retention in tumours. Their model was capable to recapitulating the major rates that determine the time course of antibody concentration within a tumour that includes: dose, affinity, plasma clearance, target expression, internalisation, permeability, and the effect of the tumour vasculature. To achieve this, they derived simple analytical expressions to 
predict the time course of drug uptake, while their model predictions were compared against relevant preclinical and clinical studies. In [88], the authors employed a 3D reaction-advection-diffusion equation to model nanoparticle flow, and used it to study the effect of flow dynamics and drug properties on nanoparticle accumulation in the liver sinusoids. To gain insight into in vivo drug delivery efficacy, [89] developed a 3D computational fluid dynamics model of continuum-scale microvascular flow and drug delivery, which was directly specified by in vivo medical imaging data from a murine mouse model. They validated their model using the in vivo data, and successfully predicted that that drug uptake by the tumour was highly heterogeneous.

\subsection{Dynamic vascular models}

Recently, dynamic models of coupled tumour growth, angiogenesis and drug delivery have emerged. While a steady-state solution is a reasonable approximation, dynamic models can simulate realistic initial conditions for a drug delivery model, such as elevated IFP as a result of tumour growth. Furthermore, dynamic models can predict the post-therapy spatio-temporal distribution of the tumour and vasculature, which is of interest for staged treatment planning. A 3D coupled model of solid tumour growth, angiogenesis and chemotherapy was proposed by [90], who employed a system of mass conservation PDEs to model oxygen, carbon dioxide, TAF and drug concentrations inside the tumour, which were dynamically coupled with a model of tumour- and angiogenesis-induced pressure. This model demonstrated that interstitial pressure affects tumour morphology via concentration gradients, and that TAF caused the greatest change in predictions in a sensitivity analysis. The authors extended this approach in [91] by coupling with the tumour 
blood and lymphatic systems, and used the model to show that lymphatic properties effect both drug concentration and retention. They also coupled drug transport with tumour growth to study the combined effects of elevated IFP and interstitial permeability on tumour regression. A 3D coupled continuum model of reaction-convection-diffusion equations was used by [92] to study the effects of dynamic network remodelling on tumour size, shape and tissue permeability on drug transport.

The preceding models considered chemo- and haptotaxis in angiogenesis, but not mechanical stimuli, which are particularly important for solid tumours where solid stresses can be highly elevated [36]. To address this, Vavourakis and his colleagues [93] developed a dynamic model of coupled solid tumour growth, extracellular matrix remodelling, angiogenesis and cytotoxic drug delivery that included a mechanotaxis term in its angiogenesis model. This allowed for a more realistic description of vascular development and architecture that was validated against in vivo murine mouse data, and hence established a biologically plausible model for drug delivery to a growing solid tumour. The model was extended by Wijeratne and Vavourakis [94] to investigate the effect of different drug sizes, such as nanoparticles, on delivery efficacy. The models showed that chemotherapeutic agents have the highest efficacy when delivered via hyper-permeable, dense vascular networks; that the time of drug administration was particularly important for low affinity drugs; and that nanoparticle distribution was highly heterogeneous.

However, while all these models have provided useful mechanistic insights into the biophysical processes governing drug delivery, they have not been quantitatively and rigorously validated against real experimental data. This 
motivates for the development of translational models that can be specified by and compared to in vivo data, which we discuss in the following section.

\section{In silico cancer models' clinical translation}

In light of the recent scientific publications reviewed in the previous sections, it is evident that the in silico cancer modelling community is rich with models [14] - with current multiscale approaches spanning from the molecular, to the cellular, up to the macroscopic level including biomechanics from a fraction of a second to months and years. Apart from their mathematical and numerical formulations, in silico models can also be categorised with respect to their complexity, the number of parameters and variables in the governing equations as well as their level of abstraction or similarity to physiologicallyor pathologically-observed behaviours. For instance, while the majority of models employ literature values for model parameters, recent efforts have been made to estimate model parameters based on imaging or experimental data. Similarly, taking advantage of the comprehensive clinical data on tumour morphology and vasculature, several recent works have focused on incorporating image-derived information into in silico cancer models, offering enhanced model fidelity and physiological similarity. Additionally, several significant contributions have been reported with a strong potential for use in surgical and radio-therapeutic treatment planning [95], to interrogate tumour normalisation strategies [96], and to test anti-cancer agents on bioartificial tissues mimicking a patient's own tissue or organ [97]. Hence, we outline below the most recent modelling works that could form the basis for in silico testing of cytotoxic agents and optimising the outcome of potential therapeu- 
tic regimens, as well as aid the repurposing of existing drugs, and design and develop novel cancer therapeutics. Importantly, such models can be used as an alternative to reduce animal testing and expedite clinical trials in humans $[98,99,100,101]$.

\subsection{In silico studies employing parameter estimation techniques}

A crucial component of model accuracy is the values selected for model parameters. Model parameters and variables usually have a biological and a physical meaning, albeit it is still exceedingly difficult (if not impossible) to quantitatively estimate some in the clinical setting. Thus, in silico cancer models often employ literature values for model parameters or select the values out of a physiological range, which creates a challenge with regards to model validation and translation.

A decade ago, Hogea and colleagues [102] presented a framework for modelling glioma growth (employing a nonlinear reaction-advection-diffusion equation) to investigate the subsequent mechanical impact on the surrounding brain tissue, i.e. mass-effect. They proposed, for the first time, an adjointbased, PDE-constrained formulation, applicable to numerically find the best set of parameters of their tumour growth model that fits patient imaging data; however, their methodology was assessed on one-dimensional numerical experiments. In the work of Liu et al. [103], a patient-specific in silico model was proposed that was specifically tailored and validated for pancreatic neuroendocrine tumour development. The model parameters were estimated by fitting model predictions to relevant clinical data through an inverse problem approach using a finite difference scheme. Their model pre-

dictions were evaluated by comparing the in silico predicted tumours against 
clinically recorded data (dual-phase CT and FDG-PET scans) on six patients with pathologically confirmed pancreatic tumours, in terms of average surface distance, tumour relative volume difference, the intracellular volume fraction map and other metrics [103]. Despite the limitations of their model, i.e. the lack of perfusion related aspects as well as the balance of oxygen and nutrients, and the use of the linear elasticity constitutive equation to describe pancreas biomechanics, the model predictions agreed very well with respect to the intracellular volume fraction (measured from dual-phase CT) and the standardised uptake value (measured from FDG-PET).

\subsection{Image-driven in silico cancer models}

As part of the continuous efforts of Stamatakos and his colleagus, a patient-specific, image-driven multiscale and multiphysics tumour simulation approach has been proposed in [104] that couples biomechanics to a cellular-level model. May et al. simulated free growth case of a small-sized primary glioblastoma multiform tumour, using a dedicated MRI head phantom, where they observed that in regions with local variations in stiffness (i.e., between different materials regions, or in regions where a significant stress is exerted) tumour cell proliferation is pronounced. In order to develop an in silico platform for simulating malignant tumour development and tissue response to treatment, the same group of investigators presented in [11] a novel approach to identify correspondence between a healthy atlas and a pathological patient image dataset. Their onco-simulator considered tumour growth in the healthy atlas using a multiscale, multiphysics model where they sought for numerical correspondence between the modified atlas and the patient MRI data via nonrigid registration. Their method appears 
promising in that it can be used for personalised in silico predictions of brain tumour development, as well as for improved atlas-based segmentation of relevant image data.

Further to this, Kolokotroni and her colleagues [12] proposed a multiscale mechanistic model to simulate lung cancer response to treatment. To inform their model they employed tumour-volumetric data of thirteen patients with non-small cell lung cancer at two time points (before and after/or during treatment: cisplatin in combination with gemcitabine, vinorelbine or docetaxel). The authors have provided a rigorous sensitivity analysis of their model, and have successfully applied it for the estimation of the efficacy of cisplatin-based doublet regimens; thus, their framework can be used for quantitative estimation of the in vivo cell-killing ability of various avenues of chemotherapy.

Recently, Angeli et al. [105] developed an in silico model of the human brain that incorporates tumour biomechanics at the tissue level and cellular events (i.e., proliferation, infiltration to surrounding tissues, and invasion to distant locations). The model was supported using anatomical magnetic resonance imaging, diffusion tensor imaging and perfusion imaging data to simulate brain tumour growth and formation of secondary nodes. This in silico study highlighted the importance of the vascular density and the features of the tumour periphery for cancerous cell infiltration and distant invasion in the brain. 


\subsection{In silico modelling of anti-angiogenic treatments and tumour normali-}

sation strategies

Jain and his colleagues have developed a series of mathematical models

of drug transport in solid tumours, pertinent for testing anti-angiogenic and ECM remodelling drugs. Stylianopoulos and Jain developed a mathematical framework for the transport of chemotherapeutic agents, which they used to investigate how to optimally combine vascular normalisation and stressalleviation treatments to improve tumour perfusion and the delivery of drugs [96]. Their paper illustrates the potential of mathematical models to simulate the normalisation of the tumour vasculature: they have demonstrated that vascular normalisation is more effective for hyperpermeable but uncompressed vessels (e.g. in glioblastomas), whereas solid stress alleviation can be more beneficial for compressed but less-permeable vessels (as in pancreatic ductal adenocarcinomas). They subsequently extended their modelling framework to account for the transport of drug-borne nanoparticles targetting cancer [106], while taking into account steric, hydrodynamic and electrostatic interactions between the nanoparticles and the vessel wall pores. The major findings of their model summarise to that electrostatic nanoparticle repulsion plays insignificant role to transvascular transport of the carriers whereas electrostatic attraction enhances their transport into the tumour interstitial space, while also, there is an optimum for the transvascular flux of negatively-charged particles with respect to their surface charge density.

Further to these works, the same group has employed mathematical models to investigate metronomic chemotherapy [107] and anti-angiogenic therapy [51]. More specifically, Mpekris and his colleagues have illustrated with 
their model that improved vessel function subsequently improves oxygenation and the delivery of drugs; thus, the presence of effector immune cells is increased whereas the number of regulatory T-cells is decreased which in turn promotes cancer cell and cancer-associated regression. Very recently, Voutouri et al. presented a mathematical model, combined with in vivo intravital microscopy imaging, and tailored to investigate the effects of hypoxia and vascular formation due to angiogenesis, as well as the kinetics of host vessel co-option of invasive glioblastoma on a mouse model. Specifically, the authors used the model to predict the spatial distribution and temporal evolution of angiogenic growth factors (with Ang2 being higher at the tumour periphery whereas VEGF being at the core of the tumour). They also tested the importance of macrophages in glioma progression, how anti-VEGF treatment can become more potent with regards to vessel co-option, and they interrogated sequential blockade of VEGF and co-option versus simultaneous blockade towards improving treatment anti-angiogenic treatment.

Boujelben and his colleagues [108], following up from the early works of Chaplain's group [109, 110], modelled blood flow, vascular permeability and diffusion within the THM to investigate the effect of these competing factors on drug delivery towards interrogating the blood brain barrier. Preliminary results of their simulations indicated that flow rate, vessel permeability and tissue diffusion coefficient interact nonlinearly, which in turn impacts drug concentration in the tumour. Also, their results suggest that under certain conditions of vascular flow and interstitial diffusion, a decrease in the blood brain barrier permeability (e.g. due to vascular normalisation therapy) may result in reduction of the drug delivered to the tumour site. The importance 
of the Boujelben et al. paper is that it demonstrates for the first time that integrating in silico and imaging models together with appropriate clinical data, here magnetic resonance and positron emission tomography images, can be used to predict drug deposition and efficacy. Via this 'Model + MRI approach,' as they entitle it, image data can be used to formulate and validate a mathematical cancer model and predict the response of a patient to a therapeutic agent.

\subsection{In silico modelling of radio/chemo-therapeutic planning}

In a parallel modelling field to the one presented in the above paragraphs, Bethge et al. [111] proposed an in silico model, CaTSiT, which encompasses the effects of chemotherapy and radiotherapy (RT): external beam radiation, radio-immunotherapy and radio-embolisation. CaTSiT was constructed using compartments and events as building blocks to mathematically model tumour progression, metastasis and response to treatment. Thus, their system can simulate tumour growth and metastatic progression under the influence of three main types of cancer therapy: primary tumour resection, chemotherapy and radiation therapy. More importantly, CaTSiT was capable to provide quantitative predictions of tumour growth and metastasis formation, and to provide comparison with clinical and experimental data.

Also, Angeli and Stylianopoulos [112] developed a three-dimensional personalised finite element model of the human brain using a biphasic tissue growth theory, to study the response of the tissue to radiation therapy. Their simulations revealed that the distribution of the mechanical forces (stresses) developing in the brain tumour and the surrounding tissue - which are crucial in tumour progression as well as efficacy of radiotherapy - is heterogeneous, 
while they found that the intratumoural fluid pressure is elevated. To the authors' opinion the predictions of the in silico model relate to clinical symptoms of brain cancers.

More recently, Gaddy and colleagues [113] modelled in silico tumourbearing mice to investigate the response to anti-angiogenic treatment with a focus to target vascular development / remodelling chemical cues, and how the response (to anti-angiogenic treatment) is influenced by the kinetics of the murine growing tumour. The authors presented a compartmental model to investigate the kinetics and transport of the vascular endothelial growth factor, which was compared and validated against reported in vivo tests in murine models. Despite the fact that their modelling methodology lacks details of the THM biomechanics or any explicit cell mechanics descriptions, this work draws a particularly useful conclusion: future in silico cancer models can use tumour kinetics, as a predictive biomarker for treatment.

In the same year, Grogan and his colleagues [114] proposed a multiscale model to predict tumour cell survival after RT, with their approach coupling a cellular automaton model of tumour growth with a model for oxygen transport from blood vessels. Their modelling work highlights that response to RT is relatively insensitive to the tumour microvascular morphology, while similar RT response predictions can be produced in relatively small tissue volumes regardless of whether realistic or artificial three-dimensional microvascular networks are employed in the models. This finding is of exceptional importance as it suggests that multiscale models of radiation therapy can produce reliable predictions of tumour regression or relapse even when pertinent vasculature (image) data to inform the model are not available. 
Finally, Agosti et al. [115] proposed a personalised model of a clinical case study of a brain tumour, employing a multiphase finite element formulation to simulate tumour growth after surgery and radiotherapy. The model was informed using relevant neuroimaging data, such as MRI and diffusion tensor image data. Although their study considered only one patient, the numerical results of the simulated tumour were in good qualitative and quantitative agreement with clinically measured tumour volume and boundaries.

\section{Discussion}

Despite the remarkable progress been made in in silico modelling in can-

cer, several important questions remain in understanding the transport of systemic drugs and nanomedicines, tumour perfusion, the treatment dynamics and cancer resistance, how to optimally combine specific chemotherapies with targeted therapies and radiation therapy. In addition, there is also a fair debate about the level of detail in silico cancer model should contain, as the more biological aspects it includes the more parameters need to be informed using in vitro, in vivo or clinical data. With the above points in mind and with the vision for in silico models becoming essential towards taming cancer and enhancing its treatment, we discuss below the main challenges in modelling the patho-physiology of the THM and its vasculature and the transport of cancer killing drugs (and in combination with other treatment modalities).

\subsection{Challenges in modelling tumour-induced angiogenesis and perfusion}

Over the last few decades, the plethora of studies on angiogenesis and tumour perfusion modelling has substantially enhanced our understanding 
of the complex interconnected processes occurring during angiogenesis along with their role in it. Importantly, angiogenesis models are a crucial link towards unravelling the mechanisms responsible for the often limited success of cancer therapeutics. However, to arrive at reliable conclusions - a prerequisite for the potential translation of in silico modelling to the clinic tumour-induced angiogenesis models need to be physiologically accurate.

Model accuracy can be radically enhanced by incorporating data-driven information into models, as well as by tuning model parameters in order to match model outcomes to patient data. For example, patient-specific characteristics such as tissue stiffness were shown to influence angiogenesis patterns along with drug delivery [93, 54]. Despite the importance of model specification to patient data, model personalisation has been limited. In particular, model personalisation has been mostly restricted to the incorporation of data-derived geometries from medical images (e.g., [116, 65, 52]) or to the specification of model parameters. For instance, a few studies have tuned the tumour growth model based on anatomical tumour images through a period of time, or estimated the tumour material parameters based on rheological data. However, in the majority of in silico studies, model parameters are based on literature values, and only subsets of them are estimated based on experimental data [51,65, 113], which could in fact come from separate datasets. Notably, angiography images of the tumour site hold significant potential to improve model specification as they could provide an initial distribution for the vasculature network. Additionally, the possibility of acquiring angiography or optical coherence tomography images of the tumour microvasculature, at different stages of cancer progression, would 
be extremely beneficial for angiogenesis models, as a means for validating the model as well as improving the accuracy of the simulated newly-formed vascular network.

With angiogenesis being a critical component of cancer models aiming in improved therapeutics, incorporating additional patient-specific characteristics $[117,118,119]$ and, thus, achieving improved accuracy in the angiogenesis modelling component should constitute a key objective of in silico modelling.

\subsection{Challenges in modelling cancer drug transport}

Similar to angiogensis modelling, the main challenge in drug delivery modelling is validation and specification (i.e. personalisation). The situation is further complicated by the different modelling regimes required to capture drug delivery (timescale order of seconds) and cell growth (timescale order of hours). Furthermore, the availability of pharmacokinetic and in vivo drug delivery data are extremely rare. Indeed, the hitherto dearth of high quality data has historically spurred the development of biophysical models, which allow predictions to be made in the absence of data (but of course in need of data to test these predictions). However, as noted by Yankeelov et al. [98], biophysical models always require some specification of material parameters, many of which are very difficult to measure in vivo. For example, a constitutive model of tissue hydro-conductivity is required by even the simplest continuum based model of drug delivery, for which tissue permeability needs to be known. In turn, permeability can depend on microstructural parameters such as collagen density and direction [120]. Therefore multi-scale biophysics underlies even the most basic models, which can rapidly increase their complexity and subsequent specification. 


\subsection{Challenges in model validation}

A fundamental prerequisite for any in silico cancer model to become a viable clinical tool, is to be thoroughly evaluated and validated in order for the clinical community to be convinced of the model's reliability and robustness. As such, a significant obstacle in the route towards clinical translation is the sparsity of validation studies for cancer models.

Currently, the majority of models provide either no validation information or are evaluated against a small subset of experimental data. This is predominantly due to the lack of suitable experimental and clinical data. For instance, validating the newly-formed vascular network as predicted through angiogenesis models constitutes a significant challenge due to the difficulty in acquiring angiographic images at different stages of tumour progression (particularly at the early stages). At the same time, most multiscale cancer models involve several variables, rendering the validation of all of them with appropriate data - especially data from the same dataset - extremely challenging. In such cases, selecting which phenomena the model should be able to replicate, or which model variables should be matched to experimental data, generates additional obstacles for model validation.

Further difficulties with model validation arise due to the challenge of defining what model validation should actually constitute. Perhaps the most obvious feature of model validation involves evaluating the model against experimental and clinical data as already discussed. Arguably, model validation could be performed by evaluating models' ability to answer or replicate biological hypotheses. This aspect of model validation is, however, very demanding as it would require experiments to be designed specifically to inves- 
tigate particular biological hypotheses. Interestingly, model validation could also be considered with respect to the agreement against clinical outcomes. For instance, one could argue that model validation could be achieved simply by a model whose outcome can reliably inform a clinical decision - without any need for assessing the accuracy of remaining model variables. Such form of model validation might perhaps be the most straightforward to achieve, as it requires the minimum amount of validation data.

With in silico modelling gaining significant ground in terms of accuracy and robustness, model validation will be increasingly necessary to enable use of cancer model applications in the clinic. Accordingly, there is an imperative need for studies to combine methodological improvements with the acquisition of appropriate validation data. Notably, studies would have to consider the type of model validation they want to achieve - preferably at the initial stage of planning the experimental and modelling work - which should partly be determined by the main objectives of each study.

\subsection{The future of in silico modelling}

Given these challenges, we argue that the best approach is to balance between model complexity and specification, using the most basic (minimum parameterised) biophysical model in conjunction with in vivo data from medical images. For example, tissue permeability can be measured accurately using diffusion-weighted (DW) MRI, which can also be combined with advanced computational modelling to extract microstructural information [121]. Given a rich enough dataset, model specification and validation can then be performed in a Bayesian framework, whereby parameters are

optimised and validated using cross-validation. Model selection can then be 
performed using likelihood-based methods, such as the Bayesian information criterion, to identify the optimal balance between model fit and complexity. Therefore, as larger datasets become available, we expect a shift from traditional first principles models to more data-driven probabilistic methods, which can exploit the strengths of biophysical models and in vivo data to yield truly personalised predictions.

In summary, and keeping in mind the importance of predicting and understanding model outcomes on a patient-specific basis, we believe that future efforts should be directed towards data-driven models. For models to be fully specified to data, model complexity might need to be considered, as existing models are often very elaborate, with a large number of unknown parameters. Instead, simpler models might be able to provide a balance between model accuracy and parameter identifiability (see for example [122, 123]). Additionally, sensitivity analysis can provide useful insight identifying the parameters that should be personalised as well as for assessing the robustness of the models to data noise. Finally, model validation should be an essential part of the modelling framework, as a means of identifying directions for enhancement of model accuracy. More importantly, model validation should be considered at the early stages of study conception, as a key step for building confidence on model outcomes amongst the clinical community, and establishing in silico modelling as a diagnostic and prognostic clinical tool.

\section{Acknowledgements}

The authors would like to acknowledge funding from the University of Cyprus. M.H. gratefully acknowledges the financial support received from 
the European Regional Development Fund and the Republic of Cyprus, as part of the RESTART 2016-2020 Programmes of the Research and Innovation Foundation of Cyprus (OPPORTUNITY/0916/MSCA/0012). P.A.W. was funded by a Medical Research Council Skills Development Fellowship (MR/T027770/1).

\section{References}

[1] M. Junttila, F. de Sauvage, Influence of tumour micro-environment heterogeneity on therapeutic response, Nature 501 (2013) 346. doi: 10.1038/nature12626.

[2] M. Wang, J. Zhao, L. Zhang, F. Wei, Y. Lian, Y. Wu, Z. Gong, S. Zhang, J. Zhou, K. Cao, X. Li, W. Xiong, G. Li, Z. Zeng, C. Guo, Role of tumor microenvironment in tumorigenesis, Journal of Cancer 8 (5) (2017) 761-773. doi:10.7150/jca.17648.

[3] C. Belli, D. Trapani, G. Viale, P. D’Amico, B. A. Duso, P. D. Vigna, F. Orsi, G. Curigliano, Targeting the microenvironment in solid tumors, Cancer Treatment Reviews 65 (2018) 22 - 32. doi:10.1016/j. ctrv.2018.02.004.

[4] M. Dewhirst, T. Secomb, Transport of drugs from blood vessels to tumour tissue, Nature Reviews Cancer 17 (2017) 738 - 750. doi: 10.1038/nrc. 2017.93 .

[5] R. Thomas, T. Van Dyke, G. Merlino, C.-P. Day, Concepts in cancer modeling: A brief history, Cancer Research (2016). doi:10.1158/ 0008-5472. CAN-16-1293. 
[6] A. Sontheimer-Phelps, B. Hassell, D. Ingber, Modelling cancer in microfluidic human organs-on-chips, Nature Reviews Cancer 19 (2) (2019) 65-81. doi:10.1038/s41568-018-0104-6.

[7] Z. Ji, K. Yan, W. Li, H. Hu, X. Zhu, Mathematical and Computational Modeling in Complex Biological Systems, BioMed research international 2017 (2017) 5958321. doi:10.1155/2017/5958321.

[8] R. Araujo, D. McElwain, A history of the study of solid tumour growth: The contribution of mathematical modelling, Bulletin of Mathematical Biology 66 (5) (2004) 1039. doi:10.1016/j.bulm.2003.11.002.

[9] A. Hoekstra, B. Chopard, P. Coveney, Multiscale modelling and simulation: a position paper, Philosophical Transactions of the Royal Society A: Mathematical, Physical and Engineering Sciences 372 (2021) (2014) 20130377. doi:10.1098/rsta.2013.0377.

[10] P. Bhattacharya, M. Viceconti, Multiscale modeling methods in biomechanics, Wiley Interdisciplinary Reviews: Systems Biology and Medicine 9 (3) (2017) e1375. doi:10.1002/wsbm.1375.

[11] S. Bauer, C. May, D. Dionysiou, G. Stamatakos, P. Buchler, M. Reyes, Multiscale modeling for image analysis of brain tumor studies, IEEE Transactions on Biomedical Engineering 59 (1) (2012) 25-29. doi: 10.1109/TBME. 2011.2163406.

[12] E. Kolokotroni, D. Dionysiou, C. Veith, Y.-J. Kim, J. Sabczynski, A. Franz, A. Grgic, J. Palm, R. Bohle, G. Stamatakos, In Silico Oncology: Quantification of the In Vivo Antitumor Efficacy of Cisplatin- 
Based Doublet Therapy in Non-Small Cell Lung Cancer (NSCLC) through a Multiscale Mechanistic Model, PLOS Computational Biology 12 (9) (2016) 1-43. doi:10.1371/journal.pcbi.1005093.

[13] D. Wu, C. Rice, X. Wang, Cancer bioinformatics: A new approach to systems clinical medicine, BMC Bioinformatics 13 (1) (2012) 71. doi:10.1186/1471-2105-13-71.

[14] G. Powathil, M. Swat, M. Chaplain, Systems oncology: Towards patient-specific treatment regimes informed by multiscale mathematical modelling, Seminars in Cancer Biology 30 (2015) 13-20, cancer modeling and network biology. doi:10.1016/j.semcancer.2014.02. 003.

[15] P. Altrock, L. Liu, F. Michor, The mathematics of cancer: integrating quantitative models, Nature Reviews Cancer 15 (2015) 730-745. doi: $10.1038 / \mathrm{nrc} 4029$.

[16] L. Edelman, J. Eddy, N. Price, In silico models of cancer, Wiley Interdisciplinary Reviews: Systems Biology and Medicine 2 (4) (2010) 438-459. doi:10.1002/wsbm.75.

[17] J. Oden, E. Lima, R. Almeida, Y. Feng, M. Rylander, D. Fuentes, D. Faghihi, M. Rahman, M. DeWitt, M. Gadde, J. Zhou, Toward predictive multiscale modeling of vascular tumor growth, Archives of Computational Methods in Engineering 23 (4) (2016) 735-779. doi:10.1007/s11831-015-9156-x. 
[18] S. Magi, K. Iwamoto, M. Okada-Hatakeyama, Current status of mathematical modeling of cancer - From the viewpoint of cancer hallmarks, Current Opinion in Systems Biology 2 (2017) 39 - 48, regulatory and metabolic networks • Cancer and systemic diseases. doi: $10.1016 / j$.coisb. 2017.02 .008 .

[19] P. Carmeliet, R. Jain, Angiogenesis in cancer and other diseases, Nature 407 (1) (2000) 249-257. doi:10.3949/ccjm.54.1.63-a.

[20] P. Carmeliet, R. Jain, Molecular mechanisms and clinical applications of angiogenesis, Nature 473 (7347) (2011) 298-307. doi:10.1016/ s0140-6736(01) 91146-8.

[21] P. Dogra, J. Butner, Y.-L. Chuang, S. Caserta, S. Goel, C. Brinker, V. Cristini, Z. Wang, Mathematical modeling in cancer nanomedicine: a review, Biomedical Microdevices 21 (2) (2019) 40. doi:10.1007/ s10544-019-0380-2.

[22] J. Folkman, Angiogenesis in cancer, vascular, rheumatoid and other disease, Nature Medicine 1 (1995) 27-31.

[23] L. Liotta, P. Steeg, W. Stetler-Stevenson, Cancer metastasis and angiogenesis: An imbalance of positive and negative regulation, Cell 64 (2) (1991) 327-336. doi:10.1016/0092-8674(91)90642-C.

[24] J. Folkman, Tumor Angiogenesis: Therapeutic Implications, The New England Journal of Medicine 285 (1971) 1182-1186. doi:10.1146/ annurev.med.57.121304.131306. 
[25] E. Ruoslahti, How Cancer Spreads, Scientific American 275 (1996) 7277.

[26] P. Carmeliet, Angiogenesis in life, disease and medicine, Nature 438 (7070) (2005) 932-936. doi:10.1038/nature04478.

[27] M. Potente, H. Gerhardt, P. Carmeliet, Basic and therapeutic aspects of angiogenesis, Cell 146 (6) (2011) 873-887. doi:10.1016/j.cell. 2011.08 .039$.

[28] D. Shweiki, A. Itin, D. Soffer, E. Keshet, Vascular endotehlial growth factor induced by hypoxia may mediate hypoxia-induced angiogenesis, Nature 359 (1992) 843-845.

[29] J. McCarthy, L. Furcht, Laminin and fibronectin promote the haptotacic migration of B16 mouse melanoma cells in vitro, Journal of Cell Biology 98 (4) (1984) 1474-1480. doi:10.1083/jcb.98.4.1474.

[30] S. Li, P. Butler, Y. Wang, Y. Hu, D. Han, S. Usami, J.-l. Guan, S. Chien, The role of the dynamics of focal adhesion kinase in the mechanotaxis of endothelial cells, Proceedings of the National Academy of Sciences 99 (6) (2002) 3546-3551.

[31] W. Risau, Mechanisms of angiogenesis, Nature 386 (April) (1997) 671674 .

[32] S. Peirce, Computational and mathematical modeling of angiogenesis, Microcirculation 15 (8) (2008) 739-751. doi:10.1080/ 10739680802220331. 
[33] R. Jain, Normalization of Tumor Vasculature: An Emerging Concept in Antiangiogenic Therapy, Science 307 (2005) 58-62. doi:10.4161/ cc. 22465 .

[34] A. S. Narang, S. Varia, Role of tumor vascular architecture in drug delivery, Advanced Drug Delivery Reviews 63 (8) (2011) 640 - 658, target Cell Movement in Tumor and Cardiovascular Diseases. doi: $10.1016 / j$. addr.2011.04.002.

[35] R. Jain, J. Martin, T. Stylianopoulos, The role of mechanical forces in tumor growth and therapy, Annu Rev Biomed Eng 16 (2014) 321-346. doi:10.1146/annurev-bioeng-071813-105259. The.

[36] T. Stylianopoulos, J. Martin, V. Chauhan, S. Jain, B. Diop-Frimpong, N. Bardeesy, B. Smith, C. Ferrone, F. Hornicek, Y. Boucher, L. Munn, R. Jain, Causes, consequences, and remedies for growth-induced solid stress in murine and human tumors, Proceedings of the National Academy of Sciences 109 (38) (2012) 15101-15108. doi:10.1073/ pnas. 1213353109.

[37] N. Mantzaris, S. Webb, H. Othmer, Mathematical modeling of tumorinduced angiogenesis, Journal of Mathematical Biology 49 (2004) 111187.

[38] M. Scianna, C. Bell, L. Preziosi, A review of mathematical models for the formation of vascular networks, Journal of Theoretical Biology 333 (2013) 174 - 209. doi:10.1016/j.jtbi.2013.04.037. 
[39] D. Balding, D. McElwain, A mathematical model of tumour-induced capillary growth, Journal of Theoretical Biology 114 (1) (1985) 53-73. doi:10.1016/S0022-5193(85)80255-1.

[40] H. Byrne, M. Chaplain, Mathematical models for tumour angiogenesis: Numerical simulations and nonlinear wave solutions, Bulletin of Mathematical Biology 57 (3) (1995) 461-486.

[41] M. Orme, M. Chaplain, Two-dimensional models of tumour angiogenesis and anti-angiogenesis strategies, Journal of Mathematics Applied in Medicine \& Biology (1997) 189-205.

[42] A. Anderson, M. Chaplain, Continuous and Discrete Mathematical Models of Tumor-induced Angiogenesis, Bulletin of Mathematical Biology 60 (1998) 857-900.

[43] M. Holmes, B. Sleeman, A Mathematical Model of Tumour Angiogenesis Incorporating Cellular Traction and Viscoelastic Effects, Journal of Theoretical Biology 202 (2) (2000) 95-112. doi:10.1006/jtbi.1999. 1038.

[44] D. Manoussaki, S. Lubkin, R. Vemon, J. Murray, A mechanical model for the formation of vascular networks in vitro, Acta Biotheoretica 44 (3) (1996) 271-282. doi:10.1007/BF00046533.

[45] J. D. Murray, On the mechanochemical theory of biological pattern formation with application to vasculogenesis, Comptes rendus biologies 326 (2) (2003) 239-252. doi:10.1016/s1631-0691(03)00065-9. 
[46] H. Levine, B. Sleeman, M. Nilsen-Hamilton, Mathematical modeling of the onset of capillary formation initiating angiogenesis, Journal of Mathematical Biology 42 (3) (2001) 195-238. doi:10.1007/ s002850000037.

[47] H. Levine, B. Sleeman, M. Nilsen-Hamilton, A mathematical model for the roles of pericytes and macrophages in the initiation of angiogenesis. I. The role of protease inhibitors in preventing angiogenesis, Mathematical Biosciences 168 (1) (2000) 77-115. doi : 10.1016/S0025-5564(00) 00034-1.

[48] E. De Angelis, L. Preziosi, Advection-Diffusion Models for Solid Tumour Evolution in Vivo and Related Free Boundary Problem, Mathematical Models and Methods in Applied Sciences 10 (03) (2000) 379407. doi:10.1142/s0218202500000239.

[49] C. Hogea, B. Murray, J. Sethian, Simulating complex tumor dynamics from avascular to vascular growth using a general level-set method, Journal of Mathematical Biology 53 (1) (2006) 86-134. doi:10.1007/ s00285-006-0378-2.

[50] J. Kremheller, A.-T. Vuong, L. Yoshihara, W. Wall, B. Schrefler, A monolithic multiphase porous medium framework for (a-)vascular tumor growth, Computer Methods in Applied Mechanics and Engineering 340 (2018) 657 - 683. doi:10.1016/j.cma.2018.06.009.

[51] C. Voutouri, N. Kirkpatrick, E. Chung, F. Mpekris, J. Baish, L. Munn, D. Fukumura, T. Stylianopoulos, R. Jain, Experimental and computa- 
tional analyses reveal dynamics of tumor vessel cooption and optimal treatment strategies, Proceedings of the National Academy of Sciences 116 (7) (2019) 2662-2671. doi:10.1073/pnas.1818322116.

[52] D. A. Hormuth, A. M. Jarrett, X. Feng, T. E. Yankeelov, Calibrating a Predictive Model of Tumor Growth and Angiogenesis with Quantitative MRI, Annals of Biomedical Engineering 47 (7) (2019) 1539-1551. doi: $10.1007 / \mathrm{s} 10439-019-02262-9$.

[53] R. Travasso, E. Poiré, M. Castro, J. Rodrguez-Manzaneque, A. Hernández-Machado, Tumor angiogenesis and vascular patterning: A mathematical model, PLoS ONE 6 (5) (2011) 1-10. doi: 10.1371/journal. pone.0019989.

[54] P. Santos-Oliveira, A. Correia, T. Rodrigues, T. Ribeiro-Rodrigues, P. Matafome, J. Rodríguez-Manzaneque, R. Seiça, H. Girão, R. Travasso, The Force at the Tip - Modelling Tension and Proliferation in Sprouting Angiogenesis, PLoS Computational Biology 11 (8) (2015) 1-20. doi:10.1371/journal.pcbi.1004436.

[55] J. Xu, G. Vilanova, H. Gomez, Full-scale, three-dimensional simulation of early-stage tumor growth: The onset of malignancy, Computer Methods in Applied Mechanics and Engineering 314 (2017) 126-146. doi:10.1016/j.cma.2016.07.010.

[56] G. Vilanova, I. Colominas, H. Gomez, Computational modeling of tumor-induced angiogenesis, Archives of Computational Meth- 
ods in Engineering 24 (4) (2017) 1071-1102. doi:10.1007/ s11831-016-9199-7.

[57] G. Vilanova, M. Burés, I. Colominas, H. Gomez, Computational modelling suggests complex interactions between interstitial flow and tumour angiogenesis, Journal of The Royal Society Interface 15 (146) (2018) 20180415. doi:10.1098/rsif.2018.0415.

[58] M. Moreira-Soares, R. Coimbra, L. Rebelo, J. Carvalho, R. Travasso, Angiogenic factors produced by hypoxic cells are a leading driver of anastomoses in sprouting angiogenesis-a computational study, Scientific Reports 8 (1) (2018) 8726. doi:10.1038/s41598-018-27034-8.

[59] S. Sun, M. Wheeler, M. Obeyesekere, C. Patrick, A deterministic model of growth factor-induced angiogenesis, Bulletin of Mathematical Biology 67 (2) (2005) 313-337. doi:10.1016/j.bulm.2004.07.004.

[60] S. Sun, M. F. Wheeler, M. Obeyesekere, C. P. Jr., Nonlinear behaviors of capillary formation in a deterministic angiogenesis model, Nonlinear Analysis: Theory, Methods \& Applications 63 (5) (2005) e2237 - e2246, invited Talks from the Fourth World Congress of Nonlinear Analysts (WCNA 2004). doi:10.1016/j.na.2005.01.066.

[61] F. Milde, M. Bergdorf, P. Koumoutsakos, A hybrid model for threedimensional simulations of sprouting angiogenesis, Biophysical Journal 95 (7) (2008) 3146-3160. doi:10.1529/biophys j.107.124511.

[62] X. Zheng, G. Koh, T. Jackson, A continuous model of angiogenesis: Initiation, extension, and maturation of new blood vessels modulated 
by vascular endothelial growth factor, angiopoietins, platelet-derived growth factor-B, and pericytes, Discrete \& Continuous Dynamical Systems - B 18 (2013) 1109. doi:10.3934/dcdsb.2013.18.1109.

[63] X. Zheng, C. Xie, A viscoelastic model of blood capillary extension and regression: Derivation, analysis, and simulation, Journal of Mathematical Biology 68 (1-2) (2014) 57-80. doi:10.1007/s00285-012-0624-8.

[64] X. Zheng, M. Sweidan, A mathematical model of angiogenesis and tumor growth: analysis and application in anti-angiogenesis therapy, Journal of Mathematical Biology 77 (5) (2018) 1589-1622. doi:10. $1007 / \mathrm{s} 00285-018-1264-4$.

[65] V. Vavourakis, P. A. Wijeratne, R. J. Shipley, M. Loizidou, T. Stylianopoulos, D. J. Hawkes, A validated multiscale in-silico model for mechano-sensitive tumour angiogenesis and growth, PLOS Computational Biology 13 (1) (2017) 1-38. doi:10.1371/journal.pcbi. 1005259.

[66] D. Dell'Orco, M. Lundqvist, C. Oslakovic, T. Cedervall, S. Linse, Modeling the time evolution of the nanoparticle-protein corona in a body fluid, PLoS One 5 (6) (2010) e10949. doi:10.1371/journal.pone. 0010949.

[67] P. Decuzzi, M. Ferrari, The receptor-mediated endocytosis of nonspherical particles, Biophysical Journal 94 (10) (2008) 3790-3797. doi:10.1529/biophysj.107.120238. 
[68] J. Siepmann, F. Siepmann, Mathematical modeling of drug delivery, International Journal of Pharmaceutics 364 (2008) 328-343. doi:10. 1016/j.ijpharm.2008.09.004.

[69] W. Zhan, M. Alamer, X. Y. Xu, Computational modelling of drug delivery to solid tumour: understanding the interplay between chemotherapeutics and biological system for optimised delivery systems, Microvascular Research 132 (2018) 81-103. doi:doi:10.1016/j.addr. 2018.07 .013$.

[70] A. Fick, Ueber diffusion, Poggendorff's Annalen der Physik 94 (1855) 59-86. doi:10.1002/andp.18551700105.

[71] F. Yuan, L. Baxter, R. Jain, Pharmacokinetic analysis of two-step approaches using bifunctional and enzyme-conjugated antibodies, Cancer Research 51 (1991) 3119-3130.

[72] L. T. Baxter, F. Yuan, R. K. Jain, Pharmacokinetic Analysis of the Perivascular Distribution of Bifunctional Antibodies and Haptens: Comparison with Experimental Data, Cancer Research 52 (1992) 58385844 .

[73] L. T. Baxter, R. K. Jain, Pharmacokinetic analysis of the microscopic distribution of enzyme-conjugated antibodies and prodrugs: comparison with experimental data, British Journal of Cancer 73 (1996) 447456.

[74] R. Jain, Physiological barriers to delivery of monoclonal antibodies and 
other macromolecules in tumors, Cancer Research 50 (3 Suppl) (1990) $814 \mathrm{~s}-819 \mathrm{~s}$.

[75] H. Byrne, M. Chaplain, Growth of nonnecrotic tumors in the presence and absence of inhibitors, Mathematical Biosciences 130 (1994) 151181.

[76] T. Jackson, H. Byrne, A mathematical model to study the effects of drug resistance and vasculature on the response of solid tumors to chemotherapy, Mathematical Biosciences 164 (2000) 17-38. doi:10. 1016/S0025-5564(99)00062-0.

[77] A. Bertuzzi, A. D’Onofrio, A. Fasano, A. Gandolfi, Regression and regrowth of tumour cords following single-dose anticancer treatment, Bulletin of Mathematical Biology 65 (2003) 903-931. doi:10.1016/ S0092-8240(03) 00054-5.

[78] J. Ward, J. King, Mathematical modelling of drug transport in tumour multicell spheroids and monolayer cultures, Mathematical Biosciences 181 (2) (2003) 177-207. doi:10.1016/S0025-5564(02)00148-7.

[79] S. McDougall, A. Anderson, M. Chaplain, J. Sherratt, Mathematical modelling of flow through vascular networks: Implications for tumourinduced angiogenesis and chemotherapy strategies, Bulletin of Mathematical Biology 64 (2002) 673-702. doi:10.1006/bulm.2002.0293.

[80] A. Stéphanou, S. McDougall, A. Anderson, M. Chaplain, Mathematical modelling of flow in $2 \mathrm{~d}$ and $3 \mathrm{~d}$ vascular networks: Applications to antiangiogenic and chemotherapeutic drug strategies, Mathematical and 
Computer Modelling 41 (2005) 1137-1156. doi:10.1016/j .mcm. 2005. 05.008 .

[81] P. Decuzzi, F. Causa, M. Ferrari, P. Netti, The effective dispersion of nanovectors within the tumor microvasculature, Annals of Biomedical Engineering 34 (4) (2006) 633-641. doi:10.1007/ s10439-005-9072-6.

[82] F. Gentile, M. Ferrari, P. Decuzzi, The transport of nanoparticles in blood vessels: the effect of vessel permeability and blood rheology, Annals of Biomedical Engineering 36 (2) (2008) 254-261. doi:10. 1007/s10439-007-9423-6.

[83] J. P. Sinek, S. Sanga, X. Zheng, H. B. Frieboes, M. Ferrari, V. Cristini, Predicting drug pharmacokinetics and effect in vascularized tumors using computer simulation, Journal of Mathematical Biology 58 (4) (2009) 485-510. doi:10.1007/s00285-008-0214-y.

[84] J. Zhao, H. Salmon, M. Sarntinoranont, Effect of heterogeneous vasculature on interstitial transport within a solid tumor, Microvascular Research 73 (2) (2007) 224-236. doi:10.1016/j.mvr.2006.12.003.

[85] K. Erbertseder, J. Reichold, B. Flemisch, P. Jenny, R. Helmig, A coupled discrete/continuum model for describing cancer-therapeutic transport in the lung, PLOS One (2012). doi:10.1371/journal.pone. 0031966.

[86] J. Tan, S. Shah, A. Thomas, H. Ou-Yang, Y. Liu, The influence of size, shape and vessel geometry on nanoparticle distribution, Mi- 
crofluidics and Nanofluidics 14 (1) (2013) 77-87. doi:10.1007/ s10404-012-1024-5.

[87] G. Thurber, K. Wittrup, A mechanistic compartmental model for total antibody uptake in tumors, Journal of Theoretical Biology 314 (2012) 57-68. doi:10.1016/j.jtbi.2012.08.034.

[88] K. M. Tsoi, S. A. MacParland, X.-Z. Ma, V. N. Spetzler, J. Echeverri, B. Ouyang, S. M. Fadel, E. A. Sykes, N. Goldaracena, J. M. Kaths, J. B. Conneely, B. A. Alman, M. Selzner, M. A. Ostrowski, O. A. Adeyi, A. Zilman, I. D. McGilvray, W. C. W. Chan, Mechanism of hard-nanomaterial clearance by the liver, Nature Materials 15 (2016) 1212-1221. doi:10.1038/nmat4718.

[89] A. d'Esposito, P. W. Sweeney, M. Ali, M. Saleh, R. Ramasawmy, T. A. Roberts, G. Agliardi, A. Desjardins, M. F. Lythgoe, R. B. Pedley, R. Shipley, S. Walker-Samuel, Computational fluid dynamics with imaging of cleared tissue and of in vivo perfusion predicts drug uptake and treatment responses in tumours, Nature Biomedical Engineering 2 (2018) 773-787. doi:10.1038/s41551-018-0306-y.

[90] L. Tang, A. L. van de Ven, D. Guo, V. Andasari, V. Cristini, K. C. $\mathrm{Li}$, X. Zhou, Computational modeling of 3d tumor growth and angiogenesis for chemotherapy evaluation, PLOS One (2014). doi: 10.1371/journal. pone. 0083962.

[91] M. Wu, H. Frieboes, M. Chaplain, S. McDougall, V. Cristini, J. Lowengrub, The effect of interstitial pressure on therapeutic agent transport: 
Coupling with the tumor blood and lymphatic vascular systems, Journal of Theoretical Biology 355 (2014) 194-207. doi:10.1016/j.jtbi. 2014.04 .012$.

[92] M. Sefidgar, M. Soltani, K. Raahemifar, H. Bazmara, S. M. M. Nayinian, B. M., Effect of tumor shape, size, and tissue transport properties on drug delivery to solid tumors, Journal of Biological Engineering 8 (12) (2014). doi:10.1186/1754-1611-8-12.

[93] V. Vavourakis, T. Stylianopoulos, P. A. Wijeratne, In-silico dynamic analysis of cytotoxic drug administration to solid tumours: Effect of binding affinity and vessel permeability, PLoS Computational Biology 14 (10) (2018) 1-30. doi:10.1371/journal.pcbi.1006460.

[94] P. Wijeratne, V. Vavourakis, A quantitative in silico platform for simulating cytotoxic and nanoparticle drug delivery to solid tumours, Interface Focus 9 (2019) 20180063. doi:10.1098/rsfs.2018.0063.

[95] G. Stamatakos, E. Kolokotroni, D. Dionysiou, E. Georgiadi, C. Desmedt, An advanced discrete state-discrete event multiscale simulation model of the response of a solid tumor to chemotherapy: Mimicking a clinical study, Journal of Theoretical Biology 266 (1) (2010) 124 - 139. doi:https://doi.org/10.1016/j.jtbi.2010.05.019.

[96] T. Stylianopoulos, R. Jain, Combining two strategies to improve perfusion and drug delivery in solid tumors, Proceedings of the National Academy of Sciences 110 (46) (2013) 18632-18637. doi:10.1073/ pnas. 1318415110. 
[97] G. Rijal, W. Li, A versatile 3d tissue matrix scaffold system for tumor modeling and drug screening, Science Advances 3 (9) (2017) e1700764e1700764. doi:10.1126/sciadv. 1700764.

[98] T. Yankeelov, G. An, O. Saut, E. Luebeck, A. Popel, B. Ribba, P. Vicini, X. Zhou, J. Weis, K. Ye, G. Genin, Multi-scale modeling in clinical oncology: Opportunities and barriers to success, Annals of Biomedical Engineering 44 (9) (2016) 2626-2641. doi:10.1007/ s10439-016-1691-6.

[99] M. Mitchell, R. Jain, R. Langer, Engineering and physical sciences in oncology: challenges and opportunities, Nature Reviews Cancer 17 (2017) 659 EP. doi:10.1038/nrc.2017.83.

[100] C. Jean-Quartier, F. Jeanquartier, I. Jurisica, A. Holzinger, In silico cancer research towards 3R, BMC Cancer 18 (1) (2018) 408. doi: $10.1186 / \mathrm{s} 12885-018-4302-0$.

[101] C. Mottini, F. Napolitano, Z. Li, X. Gao, L. Cardone, Computer-aided drug repurposing for cancer therapy: Approaches and opportunities to challenge anticancer targets, Seminars in Cancer Biology (2019). doi:10.1016/j.semcancer.2019.09.023.

[102] C. Hogea, C. Davatzikos, G. Biros, An image-driven parameter estimation problem for a reaction-diffusion glioma growth model with mass effects, Journal of Mathematical Biology 56 (6) (2008) 793-825. doi:10.1007/s00285-007-0139-x. 
[103] Y. Liu, S. Sadowski, A. Weisbrod, E. Kebebew, R. Summers, J. Yao, Patient specific tumor growth prediction using multimodal images, Medical Image Analysis 18 (3) (2014) 555 - 566. doi:10.1016/j. media.2014.02.005.

[104] C. May, E. Kolokotroni, G. Stamatakos, P. Büchler, Coupling biomechanics to a cellular level model: An approach to patient-specific image driven multi-scale and multi-physics tumor simulation, Progress in Biophysics and Molecular Biology 107 (1) (2011) 193 - 199. doi: 10.1016/j.pbiomolbio.2011.06.007.

[105] S. Angeli, K. Emblem, P. Due-Tonnessen, T. Stylianopoulos, Towards patient-specific modeling of brain tumor growth and formation of secondary nodes guided by dti-mri, NeuroImage: Clinical 20 (2018) 664673. doi:10.1016/j.nicl.2018.08.032.

[106] T. Stylianopoulos, K. Soteriou, D. Fukumura, R. Jain, Cationic Nanoparticles Have Superior Transvascular Flux into Solid Tumors: Insights from a Mathematical Model, Annals of Biomedical Engineering 41 (1) (2013) 68-77. doi:10.1007/s10439-012-0630-4.

[107] F. Mpekris, J. Baish, T. Stylianopoulos, R. Jain, Role of vascular normalization in benefit from metronomic chemotherapy, Proceedings of the National Academy of Sciences 114 (8) (2017) 1994-1999. doi:10.1073/pnas. 1700340114 .

[108] A. Boujelben, M. Watson, S. McDougall, Y.-F. Yen, E. Gerstner, C. Catana, T. Deisboeck, T. Batchelor, D. Boas, B. Rosen, J. Kalpathy- 
Cramer, M. Chaplain, Multimodality imaging and mathematical modelling of drug delivery to glioblastomas, Interface focus 6 (5) (2016) 20160039-20160039. doi:10.1098/rsfs.2016.0039.

[109] M. Chaplain, A. Stuart, A Mathematical Model for the Diffusion of Tumour Angiogenesis Factor into the Surrounding Host Tissue, Mathematical Medicine and Biology: A Journal of the IMA 8 (3) (1991) 191-220. doi:10.1093/imammb/8.3.191.

[110] M. A. J. Chaplain, Mathematical Modelling of angiogenesis, Journal of neuro-oncology 50 (2000) 37-51. doi:10.1023/A:1006446020377.

[111] A. Bethge, U. Schumacher, G. Wedemann, Simulation of metastatic progression using a computer model including chemotherapy and radiation therapy, Journal of Biomedical Informatics 57 (2015) 74-87. doi:10.1016/j.jbi.2015.07.011.

[112] S. Angeli, T. Stylianopoulos, Biphasic modeling of brain tumor biomechanics and response to radiation treatment, Journal of Biomechanics 49 (9) (2016) 1524-1531. doi:10.1016/j.jbiomech.2016.03.029.

[113] T. Gaddy, Q. Wu, A. Arnheim, S. Finley, Mechanistic modeling quantifies the influence of tumor growth kinetics on the response to antiangiogenic treatment, PLOS Computational Biology 13 (12) (2017) 1-23. doi:10.1371/journal.pcbi.1005874.

[114] J. A. Grogan, B. Markelc, A. J. Connor, R. J. Muschel, J. M. PittFrancis, P. K. Maini, H. M. Byrne, Predicting the influence of microvascular structure on tumor response to radiotherapy, IEEE Transactions 
on Biomedical Engineering 64 (3) (2017) 504-511. doi:10.1109/TBME. 2016.2606563.

[115] A. Agosti, C. Giverso, E. Faggiano, A. Stamm, P. Ciarletta, A personalized mathematical tool for neuro-oncology: A clinical case study, International Journal of Non-Linear Mechanics 107 (2018) 170 - 181. doi:10.1016/j.ijnonlinmec.2018.06.004.

[116] H. Perfahl, H. Byrne, T. Chen, V. Estrella, T. Alarcón, A. Lapin, R. Gatenby, R. Gillies, M. Lloyd, P. Maini, M. Reuss, M. Owen, Multiscale modelling of vascular tumour growth in 3d: The roles of domain size and boundary conditions, PLOS ONE 6 (4) (2011) 1-17. doi:10.1371/journal.pone. 0014790.

[117] J. Laufer, E. Zhang, B. Treeby, B. Cox, P. Beard, P. Johnson, B. Pedley, In vivo preclinical photoacoustic imaging of tumor vasculature development and therapy, Journal of Biomedical Optics 17 (5) (2012) 1 - 9 . doi:10.1117/1. JBO.17.5.056016.

URL https://doi.org/10.1117/1.JBO.17.5.056016

[118] B. Vakoc, D. Fukumura, R. Jain, B. Bouma, Cancer imaging by optical coherence tomography: preclinical progress and clinical potential, Nature Reviews Cancer volume 12 (2012) 363-368. doi: $10.1038 / \mathrm{nrc3} 235$.

[119] A. Maslennikova, M. Sirotkina, A. Moiseev, E. Finagina, S. Ksenofontov, G. Gelikonov, L. Matveev, E. Kiseleva, V. Zaitsev, E. Zagaynova, F. Feldchtein, N. Gladkova, A. Vitkin, In-vivo longitudinal 
imaging of microvascular changes in irradiated oral mucosa of radiotherapy cancer patients using optical coherence tomography, Scientific Reports 7 (1) (2017) 16505. doi:10.1038/s41598-017-16823-2.

[120] P. Wijeratne, J. Hipwell, D. Hawkes, T. Stylianopoulos, V. Vavourakis, Multiscale biphasic modelling of peritumoural collagen microstructure: The effect of tumour growth on permeability and fluid flow, PLoS ONE (2018). doi:10.1371/journal.pone.0184511.

[121] H. Zhang, T. Schneider, C. A. Wheeler-Kingshott, D. C. Alexander, NODDI: practical in vivo neurite orientation dispersion and density imaging of the human brain, Neuroimage 61 (4) (2012) 1000-1016. doi:10.1016/j.neuroimage.2012.03.072.

[122] M. Hadjicharalambous, R. Chabiniok, L. Asner, E. Sammut, J. Wong, G. Carr-White, J. Lee, R. Razavi, N. Smith, D. Nordsletten, Analysis of passive cardiac constitutive laws for parameter estimation using 3D tagged MRI, Biomechanics and Modeling in Mechanobiology 14 (4) (2014) 807-828. doi:10.1007/s10237-014-0638-9.

[123] K. Wong, R. Summers, E. Kebebew, J. Yao, Tumor Growth Prediction with Hyperelastic Biomechanical Model, Physiological Data Fusion, and Nonlinear Optimization, in: P. Golland, N. Hata, C. Barillot, J. Hornegger, R. Howe (Eds.), Medical Image Computing and Computer-Assisted Intervention - MICCAI 2014, Springer International Publishing, 2014, pp. 25-32. doi:10.1007/ 978-3-319-10470-6_4. 